\title{
Limitations on the expressive power of convex cones without long chains of faces
}

\author{
James Saunderson*
}

August 6, 2019

\begin{abstract}
A convex optimization problem in conic form involves minimizing a linear functional over the intersection of a convex cone and an affine subspace. In some cases, it is possible to replace a conic formulation using a certain cone, with a 'lifted' conic formulation using another cone that is higher-dimensional, but simpler, in some sense. One situation in which this can be computationally advantageous is when the higher-dimensional cone is a Cartesian product of many 'low-complexity' cones, such as second-order cones, or small positive semidefinite cones.

This paper studies obstructions to a convex cone having a lifted representation with such a product structure. The main result says that whenever a convex cone has a certain neighborliness property, then it does not have a lifted representation using a finite product of cones, each of which has only short chains of faces. This is a generalization of recent work of Averkov ('Optimal size of linear matrix inequalities in semidefinite approaches to polynomial optimization', SIAM J. Appl. Alg. Geom., Vol. 3, No. 1, 128-151, 2019) which considers only lifted representations using products of positive semidefinite cones of bounded size. Among the consequences of the main result is that various cones related to nonnegative polynomials do not have lifted representations using products of 'low-complexity' cones, such as smooth cones, the exponential cone, and cones defined by hyperbolic polynomials of low degree.
\end{abstract}

\section{Introduction}

A conic optimization problem has the form

$$
\operatorname{minimize}_{x}\langle c, x\rangle \quad \text { subject to } A(x)=b, x \in K
$$

where $K \subseteq \mathbb{R}^{n}$ is a closed convex cone, $A: \mathbb{R}^{n} \rightarrow \mathbb{R}^{m}$ is a linear map, and $c \in \mathbb{R}^{n}$ and $b \in \mathbb{R}^{m}$. Different choices of convex cones in Eq. (1) lead to different classes of convex optimization problems. For instance, if $K=\mathbb{R}_{+}^{n}$ is a nonnegative orthant, we obtain a linear program; if $K$ is a finite Cartesian product of second-order cones $\mathcal{Q}=\left\{(x, y, z) \in \mathbb{R}^{3}: \sqrt{x^{2}+y^{2}} \leq z\right\}$, we obtain a secondorder cone program; if $K=\mathcal{S}_{+}^{k}$ is the cone of $k \times k$ positive semidefinite matrices, we obtain a semidefinite program. Computationally, conic optimization problems are often easier to solve if $K=K_{1} \times \cdots \times K_{m}$ where each of the $K_{i}$ are convex cones of 'low-complexity'. One reason for this is that basic algorithmic primitives related to the cone, such as computing the Euclidean projection of a point onto the cone, are separable across the factors and so are easily parallelizable.

It is natural, then, to try to understand which families of convex optimization problems can be expressed in conic form with respect to a finite Cartesian product of 'low-complexity' cones.

\footnotetext{
${ }^{*}$ Department of Electrical and Computer Systems Engineering, Monash University, VIC 3800, Australia. Email: james.saunderson@monash.edu
} 
The question can be phrased in geometric terms by using the notion of a lifted representation of a convex cone.

Definition 1.1 ([GPT13]). If $C \subseteq \mathbb{R}^{n}$ and $K \subseteq \mathbb{R}^{d}$ are closed convex cones then $C$ has a $K$-lift if $C=\pi(K \cap L)$ where $\pi: \mathbb{R}^{d} \rightarrow \mathbb{R}^{n}$ is a linear map and $L \subseteq \mathbb{R}^{d}$ is a linear subspace.

If $C$ has a $K$-lift, then any conic optimization problem using the cone $C$ can be reformulated as a conic optimization problem using $K$. As such we are interested in understanding when a convex cone $C$ has a $K$-lift where $K$ is a Cartesian product of 'low-complexity' cones. There are many examples where such reformulations are possible.

- If $C$ is the set of symmetric positive semidefinite matrices that are sparse with respect to a chordal graph, then $C$ has a $\mathcal{S}_{+}^{k_{1}} \times \cdots \times \mathcal{S}_{+}^{k_{\ell}}$-lift where the $k_{i}$ are the sizes of the maximal cliques in the graph [AHMR88, GJSW84]. This observation can be exploited to yield significant computational savings for semidefinite programs with sparse data (see, e.g., [VA15]).

- The cone of scaled diagonally dominant matrices [AM19], and the cone of sums of nonnegative circuit polynomials [IDW16], both have second-order cone lifts, or equivalently, $\left(\mathcal{S}_{+}^{2}\right)^{m}$-lifts for some $m .^{1}$ These have been used to give more tractable certificates of nonnegativity for multivariate polynomials than those given by general sums of squares.

- Ben-Tal and Nemirovski's book [BTN01] has many nontrivial examples of convex sets that have second-order cone lifts.

- Cones of sums of arithmetic-mean geometric-mean functions (SAGE functions) [CS16], used to certify nonnegativity of signomial functions, have lifted representations using a product of three-dimensional relative entropy cones (or, equivalently, exponential cones).

This paper, on the other hand, explores limitations on the expressive power of lifts using finite products of 'low-complexity' cones. Until recently, the only result in this direction was the fact that a convex cone has an $\mathbb{R}_{+}^{m}$-lift for some finite $m$ if and only if it is a polyhedral cone.

In a breakthrough paper, Fawzi [Faw18] studied the expressive power of second-order cone programming. Among his results is that $\mathcal{S}_{+}^{3}$ has no second-order cone lift. Fawzi identified the importance of certain neighborliness properties of the positive semidefinite cone as an obstruction to constructing second-order cone lifts. He also introduced a combinatorial approach to establish results of this type, via Turán's theorem.

Averkov [Ave19] subsequently significantly extended Fawzi's approach. He introduced the semidefinite extension degree of a convex cone - the smallest $k$ such that $C$ has an $\left(\mathcal{S}_{+}^{k}\right)^{m}$-lift for some positive integer $m$. Averkov established that convex cones with a certain $k$-neighborliness property (made precise in Definition 1.2) have semidefinite extension degree at least $k+1$. To prove this result, Averkov used Ramsey's theorem for uniform hypergraphs to provide the key combinatorial obstruction.

The present work moves beyond studying lifts using products of positive semidefinite cones of bounded size. Instead, we consider a notion of 'low-complexity' that just depends on the face lattice of a cone. In particular, we consider the expressive power of $K$-lifts where $K$ is a finite product of cones, each of which has only short chains of faces. The main result (Theorem 1.4) gives an explicit obstruction to a cone having a lifted representation of this type. The obstruction is the same as that considered by Averkov, and is based on the existence of arbitrarily large finite collections of extreme rays of the cone that satisfy a certain neighborliness property (see Definition 1.2).

\footnotetext{
${ }^{1}$ The fact that sums of nonnegative circuit polynomials have $\left(\mathcal{S}_{+}^{2}\right)^{m}$-lifts was established by Averkov [Ave19].
} 


\subsection{Chains of faces, neighborliness, and our main result}

\subsubsection{Chains of faces}

If $K$ is a closed convex cone, then a subset $F \subseteq K$ is a face if $x, y \in K$ and $\alpha x+(1-\alpha) y \in F$ for some $\alpha \in(0,1)$ implies that $x, y \in F$. Note that the empty set is always a face. A collection of faces $F_{1}, F_{2}, \ldots, F_{\ell} \subseteq K$ is a chain of length $\ell$ if $F_{1} \subsetneq F_{2} \subsetneq \cdots \subsetneq F_{\ell}$. For a convex cone $K$, define

$$
\ell(K)=\text { maximum length of a chain of nonempty faces of } K \text {. }
$$

This is well-defined because $\ell(K) \leq \operatorname{dim}(K)+1$ where $\operatorname{dim}(K)$ is the dimension of the span of $K$ (see Section 2.2). Crucially, $\ell(\cdot)$ is monotone, in the sense that if $F$ is a face of $K$ and $F \subsetneq K$ then $\ell(F)<\ell(K)$.

This quantity appears naturally in the study of facial reduction algorithms for conic optimization problems (see, e.g., [Pat13, WM13]), and has been used to give an upper bound on the Carathéodory number of a closed, pointed convex cone [IL17]. In this paper we will think of cones without any long chains of faces as being of low complexity. Indeed $\ell(K)=1$ if and only if $K$ is a linear subspace, and $\ell(K)=2$ if and only if $K$ is a closed halfspace.

\subsubsection{Neighborliness properties}

In Definition 1.2, to follow, we describe the cones for which we can show the non-existence of lifts. This is the same class of convex cones that is considered by Averkov [Ave19]. In the following definition, and throughout, we fix an inner product $\langle\cdot, \cdot\rangle$ on $\mathbb{R}^{n}$ and denote the associated norm by $\|\cdot\|$. If $C \subseteq \mathbb{R}^{n}$ is a set, then $C^{*}=\{f:\langle f, x\rangle \geq 0$ for all $x \in C\}$ is the corresponding dual cone. A closed convex cone $C \subseteq \mathbb{R}^{n}$ is pointed if $C \cap(-C)=\{0\}$ and full-dimensional if $\operatorname{span}(C)=\mathbb{R}^{n}$. A convex cone is proper if it is closed, pointed, and full-dimensional. For a convex cone $C \subseteq \mathbb{R}^{n}$, we denote the set of extreme rays of $C$ by $\operatorname{Ext}(C)$ and the set of normalized extreme rays by $\operatorname{ext}(C):=\left\{v \in \mathbb{R}^{n}:\|v\|=1, \mathbb{R}_{+} v \in \operatorname{Ext}(C)\right\}$.

Definition 1.2. Let $C$ be a proper convex cone. If $V \subseteq \operatorname{ext}(C)$ is a subset of normalized extreme rays of $C$, then $C$ is $k$-neighborly with respect to $V$ if for every $k$-element subset $W \subset V$, there is some linear functional $f_{W} \in C^{*}$ such that $\left\langle f_{W}, v\right\rangle>0$ if $v \in V \backslash W$ and $\left\langle f_{W}, v\right\rangle=0$ if $v \in W$.

In this paper we will be particularly interested in the case where $C$ is $k$-neighborly with respect to arbitrarily large finite subsets of normalized extreme rays of $C$.

Example 1.3 (Positive semidefinite cone). The cone of $(k+1) \times(k+1)$ positive semidefinite symmetric matrices is $k$-neighborly with respect to

$$
V=\left\{v_{i} v_{i}^{T} /\left\|v_{i}\right\|^{2}: i \in \mathbb{N}\right\} \quad \text { where } \quad v_{i}:=\left(1, i, i^{2}, \ldots, i^{k}\right) .
$$

To see why this is true, for each set $W$ of $k$ natural numbers, define the non-negative polynomial $p_{W}$ and the vector $c(W) \in \mathbb{R}^{k+1}$ such that

$$
p_{W}(t)=\left[\prod_{i \in W}(t-i)\right]^{2}=\left(\sum_{j=0}^{k} c(W)_{j} t^{j}\right)^{2}=\operatorname{tr}\left(c(W) c(W)^{T} v_{t} v_{t}^{T}\right) .
$$

Observe that $p_{W}(t)$ vanishes if and only if $t \in W$. Consequently $c(W) c(W)^{T} \in\left(\mathcal{S}_{+}^{k+1}\right)^{*}=\mathcal{S}_{+}^{k+1}$ vanishes on $v_{i} v_{i}^{T}$ if and only if $i \in W$. 
We are now in a position to state our main result.

Theorem 1.4. Let $C$ be a proper convex cone, let $m$ be a positive integer, and let $K_{1}, \ldots, K_{m}$ be closed convex cones with $\ell\left(K_{i}\right) \leq k+1$ for each $i=1,2, \ldots, m$. Suppose that, for each $N \in \mathbb{N}$ there exists a subset $V_{N} \subseteq \operatorname{ext}(C)$ such that $C$ is $k$-neighborly with respect to $V_{N}$ and $\left|V_{N}\right| \geq N$. Then $C$ does not have a $K_{1} \times \cdots \times K_{m}$-lift.

We remark that if a proper convex cone $C$ is $k$-neighborly with respect to an infinite set $V \subseteq \operatorname{ext}(C)$, then $C$ is $k$-neighborly with respect to any finite subset of $V$, and so the neighborliness hypothesis of Theorem 1.4 holds.

Section 2.1 gives examples of convex cones that are $k$-neighborly with respect to arbitrarily large finite sets of normalized extreme rays. Section 2.2 gives examples of convex cones with only short chains of faces. Combining these allows us to specialize Theorem 1.4 to produce a range of irrepresentability results. The following statements about the non-existence of lifts of certain positive semidefinite cones are examples of the kind of results that follow from Theorem 1.4:

- $\mathcal{S}_{+}^{3}$ has no $K$-lift where $K$ is a finite Cartesian product of smooth convex cones (such as second-order cones);

- $\mathcal{S}_{+}^{4}$ has no $K$-lift where $K$ is a finite Cartesian product of three-dimensional convex cones (such as cones over the epigraphs of univariate convex functions);

- $\mathcal{S}_{+}^{k+1}$ has no $\left(\mathcal{S}_{+}^{k}\right)^{m}$-lift where $m$ is a positive integer, a result from [Ave19];

- $\mathcal{S}_{+}^{k+1}$ has no $K$-lift where $K$ is a hyperbolicity cone corresponding to a hyperbolic polynomial with all its irreducible factors having degree at most $k$.

Our proof of Theorem 1.4 follows Averkov's approach to lower-bounding the semidefinite extension degree. In fact, the underlying combinatorial part of the argument is exactly the same. The main new contribution is that all of the algebraic structure of the positive semidefinite cone used by Averkov can be done away with, and replaced with basic properties of face lattice of convex cones.

\subsection{Notation}

For a convex cone $C$, let relint $(C)$ denote the relative interior of $C$. If $S \subseteq \mathbb{R}^{n}$ let cone $(S)$ be the cone generated by $S$, i.e., the collection of all non-negative combinations of elements of $S$. If $n$ is a positive integer let $[n]:=\{1,2, \ldots, n\}$. If $S$ is a finite set let $\left(\begin{array}{l}S \\ n\end{array}\right)$ be the collection of $n$-element subsets of $S$. Other notation, needed only for the proofs, will be introduced in Section 3.

\subsection{Outline}

The rest of the paper is structured as follows. In Section 2 we discuss the consequences of Theorem 1.4. We state a number of examples (many from Averkov's work) of cones that are $k$-neighborly with respect to an infinite set. We then give bounds on the length of chains of faces for a number of families of convex cones. From the discussion one can extract many irrepresentability results. For the sake of brevity we will not exhaustively state such results. In Section 3 we briefly introduce some of the technical tools used in the proof of the main result. In Section 4 we generalize the key technical results of [Ave19] to our setting, prove these results, and consequently complete the proof of Theorem 1.4. 


\section{Consequences of the main result}

To appreciate the consequences of our main result, this section is devoted to giving examples of convex cones to which Theorem 1.4 can be applied. In Section 2.1 we give examples of convex cones that are $k$-neighborly (for some $k$ ) with respect to arbitrarily large finite sets of normalized extreme rays. These are the convex cones that can be used as $C$ in Theorem 1.4. In Section 2.2 we give upper bounds on $\ell(\cdot)$, for many convex cones. These are the cones that can be used as the $K_{i}$ in Theorem 1.4.

\subsection{Cones $k$-neighborly with respect to arbitrarily large finite sets}

\subsubsection{Non-polyhedral cones}

If $C$ is a proper, non-polyhedral cone then it has infinitely many extreme rays. Moreover, it has infinitely many exposed extreme rays (extreme rays that can be obtained as the intersection of $C$ with a hyperplane), because exposed extreme rays are dense in the set of all extreme rays by Straszewicz's theorem [Roc15, Theorem 18.6]. It follows that $C$ is 1-neighborly with respect to the (infinite) set of normalized exposed extreme rays.

\subsubsection{Cones related to nonnegative polynomials and sums of squares}

A number of interesting examples are special cases of the following result, which is essentially [Ave19, Corollary 3].

Proposition 2.1. Let $X \subseteq \mathbb{R}^{n}$ have nonempty interior. Define

$$
\begin{aligned}
P_{n, 2 d}(X) & :=\{\text { polynomials } p \text { of degree } \leq 2 d \text { such that } p(x) \geq 0 \text { for all } x \in X\} \\
\Sigma_{n, 2 d} & :=\{\text { polynomials of degree } \leq 2 d \text { in } n \text { variables that are sums of squares }\} .
\end{aligned}
$$

Let $C$ be a closed convex cone that satisfies $P_{n, 2 d}(X)^{*} \subseteq C \subseteq \Sigma_{n, 2 d}^{*}$. Then for each $N \in \mathbb{N}$ there is a set $V \subseteq \operatorname{ext}(C)$ with $|V| \geq N$ such that $C$ is $\left(\left(\begin{array}{c}n+d \\ d\end{array}\right)-1\right)$-neighborly with respect $V$.

From Proposition 2.1 we can construct many examples. Perhaps the simplest, which are also discussed in Averkov's paper, are the following:

- The (self-dual) cone of $k \times k$ positive semidefinite matrices $\mathcal{S}_{+}^{k}$ is linearly isomorphic to $\Sigma_{k-1,2}$, since a quadratic polynomial in $k-1$ variables is nonnegative if and only if it has the form

$$
q(x)=\left[\begin{array}{ll}
1 & x^{T}
\end{array}\right] Q\left[\begin{array}{l}
1 \\
x
\end{array}\right]
$$

where $Q$ is positive semidefinite. It then follows from Proposition 2.1 that $\mathcal{S}_{+}^{k}$ is $(k-1)$ neighborly with respect to arbitrarily large finite subsets of normalized extreme rays. We gave a direct argument that $\mathcal{S}_{+}^{k}$ is $(k-1)$-neighborly with respect to an infinite subset of extreme rays in Example 1.3.

- The dual of the cone of univariate nonnegative polynomials of degree at most $2 d$, i.e., $P_{1,2 d}(\mathbb{R})^{*}$ is $d$-neighborly with respect to arbitrarily large finite subsets of normalized extreme rays. 


\subsubsection{Cones over $k$-neighborly manifolds}

If $\mathcal{M} \subseteq \mathbb{R}^{m}$ is a smooth embedded manifold, Kalai and Widgerson [KW08] say that $\mathcal{M}$ is $k$ neighborly if, for every subset $X \subseteq \mathcal{M}$ of cardinality $k$, there exists a linear functional $f$, and a real number $b$, such that $\langle f, x\rangle=b$ for all $x \in X$ and $\langle f, x\rangle>b$ for all $x \in \mathcal{M} \backslash X$. If $\mathcal{M}$ is a $k$-neighborly manifold of positive dimension, then $\operatorname{cone}(\mathcal{M} \times\{1\}) \subseteq \mathbb{R}^{m+1}$ is $k$-neighborly with respect to the infinite set $\{v /\|v\|: v \in \mathcal{M} \times\{1\}\}$ of normalized extreme rays.

\subsubsection{Cones with neighborly faces and derivative relaxations of $\mathcal{S}_{+}^{k}$}

Suppose that $C$ is a convex cone and $F$ is an exposed face of $C$. One can show that if $F$ is $k$ neighborly with respect to $V \subseteq \operatorname{ext}(F) \subseteq \operatorname{ext}(C)$ then $C$ is also $k$-neighborly with respect to $V$. This simple observation gives some interesting additional examples beyond cones related to nonnegative polynomials. We describe just one example here, which is an example of a hyperbolicity cone (a class of convex cones we discuss more at the end of Section 2.2).

The cone of $k \times k$ positive semidefinite matrices can be described as

$$
\mathcal{S}_{+}^{k}=\left\{X \in \mathcal{S}^{k}: E_{1, k}(X) \geq 0, E_{2, k}(X) \geq 0, \ldots, E_{k, k}(X) \geq 0\right\}
$$

where $E_{\ell, k}(X)$ is the sum of the $\ell \times \ell$ principal minors of the $k \times k$ symmetric matrix $X$ (see, e.g., [Ren06]). For $0 \leq \ell \leq k-1$ one can define a cone (which turns out to be convex) by keeping only the first $k-\ell$ of these inequalities:

$$
\mathcal{S}_{+}^{k,(\ell)}=\left\{X \in \mathcal{S}^{k}: E_{1, k}(X) \geq 0, \ldots, E_{k-\ell, k}(X) \geq 0\right\} .
$$

These are often called the derivative relaxations or Renegar derivatives of the positive semidefinite cone. For the connection to derivatives, and the fact that these are convex cones, see [Ren06]. Consider the intersection of $\mathcal{S}_{+}^{k,(\ell)}$ with the subspace $L$ of symmetric matrices of the form $\left[\begin{array}{cc}Y & 0 \\ 0 & 0\end{array}\right]$ where only the top $(k-\ell) \times(k-\ell)$ block is nonzero. Restricted to this subspace we have that

$$
E_{p, k}\left(\left[\begin{array}{cc}
Y & 0 \\
0 & 0
\end{array}\right]\right)= \begin{cases}E_{p, k-\ell}(Y) & \text { if } p \leq k-\ell \\
0 & \text { otherwise. }\end{cases}
$$

As such $\mathcal{S}_{+}^{k,(\ell)} \cap L$ is a face of $\mathcal{S}_{+}^{k,(\ell)}$ that is linearly isomorphic to $\mathcal{S}_{+}^{k-\ell}$. Since $\mathcal{S}_{+}^{k-\ell}$ is $(k-\ell-1)$ neighborly with respect to an infinite set of normalized extreme rays, it follows that $\mathcal{S}_{+}^{k,(\ell)}$ has the same property.

\subsection{Cones with only short chains of faces}

The innovation of Theorem 1.4 is that it rules out $K$-lifts where $K$ is a finite Cartesian product of cones, each of which has only short chains of faces. In this section we now give a number of examples of cones $K$ that have only short chains of faces.

\subsubsection{Rays}

The ray $\mathbb{R}_{+}$has two nonempty faces: $\{0\}$ and $\mathbb{R}_{+}$itself. It follows that $\ell\left(\mathbb{R}_{+}\right)=2$. From Theorem 1.4 we recover the fact that any cone that is 1-neighborly with respect to an infinite set of normalized extreme rays (i.e., a cone with infinitely many exposed extreme rays) cannot have a $\mathbb{R}_{+}^{m}$-lift. 


\subsubsection{Smooth cones}

Following [LP18], for instance, we call a pointed closed convex cone $K$ smooth if its only non-empty faces are $\{0\}$ or $K$ or its extreme rays. As such, any smooth cone has $\ell(K) \leq 3$. For example, the second-order cone

$$
\mathcal{Q}_{n+1}=\left\{\left(x_{0}, \ldots, x_{n}\right) \in \mathbb{R}^{n+1}: \sqrt{x_{1}^{2}+\cdots+x_{n}^{2}} \leq x_{0}\right\}
$$

is a smooth cone. From Theorem 1.4 we can conclude that any cone 2-neighborly with respect to arbitrarily large finite sets of normalized extreme rays does not have a lift using a finite product of smooth cones.

\subsubsection{Low-dimensional cones}

Since the dimension strictly increases along chains of faces [Roc15, Corollary 18.1.3], we always have that

$$
\ell(K) \leq \operatorname{dim}(K)+1 .
$$

For example, the exponential cone $K=\operatorname{cl}\left\{(x, t, y) \in \mathbb{R}^{2} \times \mathbb{R}_{++}: y e^{x / y} \leq t\right\}$ satisfies $\ell(K) \leq 3+1$. More generally, if $K=\operatorname{cl}\left\{(x, t, y) \in \mathbb{R}^{2} \times \mathbb{R}_{++}: y g(x / y) \leq t\right\}$ is the closure of the cone over the epigraph of any univariate convex function $g$, then $\ell(K) \leq 3+1$. From Theorem 1.4 we can conclude that any cone $k$-neighborly with respect to arbitrarily large finite sets of normalized extreme rays does not have a lift using a finite product of $k$-dimensional cones.

\subsubsection{The positive semidefinite cone}

The rank of a symmetric matrix is constant on the relative interior of faces of the positive semidefinite cone. Moreover, the rank function strictly increases on chains of faces. As such,

$$
\ell\left(\mathcal{S}_{+}^{k}\right) \leq k+1
$$

On the other hand, it is easy to construct a chain of non-empty faces of length $k+1$, so we have that $\ell\left(\mathcal{S}_{+}^{k}\right)=k+1$. Theorem 1.4, in this context, recovers Averkov's result that if $C$ is $k$-neighborly with respect to arbitrarily large finite sets of normalized extreme rays then $C$ has no $\left(\mathcal{S}_{+}^{k}\right)^{m}$-lift.

\subsubsection{Hyperbolicity cones}

A class of convex cones that generalizes the positive semidefinite cone are hyperbolicity cones. A homogeneous polynomial $p$ of degree $d$ in $n$ variables with real coefficients is called hyperbolic with respect to $e \in \mathbb{R}^{n}$ if $p(e)>0$ and, for all $x \in \mathbb{R}^{n}$, the univariate polynomial $t \mapsto p(t e-x)$ has only real roots. We call these real roots the hyperbolic eigenvalues of $x$. There is an associated closed cone

$$
\Lambda_{+}(p, e)=\left\{x \in \mathbb{R}^{n}: \text { all hyperbolic eigenvalues of } x \text { are nonnegative }\right\}
$$

which is convex, a result of Gårding [Går59]. As an example, the determinant restricted to symmetric matrices is hyperbolic with respect to the identity matrix, and the associated hyperbolicity cone is the positive semidefinite cone.

With a hyperbolic polynomial, one can associate a rank function by defining

$$
\operatorname{rank}_{p, e}(x)=\# \text { non-zero hyperbolic eigenvalues of } x \text {. }
$$


Renegar [Ren06, Theorem 26] has showed that if $F$ is a face of a hyperbolicity cone $\Lambda_{+}(p, e)$ then every point in the relative interior of $F$ has the same hyperbolic rank, and every point in the boundary of $F$ has strictly smaller rank. Consequently, there is a well defined notion of the hyperbolic rank of a face, and the hyperbolic rank function is strictly increasing along chains of faces. Since any point in the relative interior of the hyperbolicity cone has rank $d=\operatorname{degree}(p)$, we have the bound

$$
\ell\left(\Lambda_{+}(p, e)\right) \leq d+1
$$

The following gives a natural sufficient condition under which this bound is tight.

Proposition 2.2. Suppose that $p$ is homogeneous of degree d, hyperbolic with respect to $e$, and the associated hyperbolicity cone $\Lambda_{+}(p, e)$ is pointed. If all of the extreme rays of $\Lambda_{+}(p, e)$ have hyperbolic rank one then $\ell\left(\Lambda_{+}(p, e)\right)=d+1$.

Proof. Let $x_{1}, x_{2}, \ldots, x_{\kappa}$ be a collection of generators of extreme rays such that $x_{1}+x_{2}+\cdots+x_{\kappa}$ is in the relative interior of $\Lambda_{+}(p, e)$. Since the Carathéodory number of $\Lambda_{+}(p, e)$ is bounded above by $\ell\left(\Lambda_{+}(p, e)\right)-1$ [IL17], it follows that we can choose $\kappa \leq \ell\left(\Lambda_{+}(p, e)\right)-1$. Since the hyperbolic rank function is a nonnegative submodular function on the face lattice of the hyperbolicity cone [AB18], the hyperbolic rank is subadditive. Then

$$
\ell\left(\Lambda_{+}(p, e)\right)-1 \leq d=\operatorname{rank}_{p, e}\left(\sum_{i=1}^{\kappa} x_{i}\right) \leq \sum_{i=1}^{\kappa} \operatorname{rank}_{p, e}\left(x_{i}\right)=\kappa \leq \ell\left(\Lambda_{+}(p, e)\right)-1 .
$$

Symmetric cones $^{2}$ are examples of hyperbolicity cones with $p$ being the determinant associated with the appropriate Euclidean Jordan algebra. For these cones, all of the extreme rays have hyperbolic rank one. As such Proposition 2.2 generalizes [IL17, Theorem 14]. It also applies to spectrahedra with all rank one extreme rays which, in the real symmetric case, have been classified by Blekherman, Sinn, and Velasco [BSV17].

We can obtain a more refined bound in cases where all the extreme rays have rank at least $r$, and so all nonzero faces have rank at least $r$. Then

$$
\ell\left(\Lambda_{+}(p, e)\right) \leq d-r+2
$$

since, in that case, there are no faces of rank $1,2, \ldots, r-1$. For hyperbolicity cones corresponding to strictly hyperbolic polynomials [Nui69] (which give rise to smooth hyperbolicity cones), all extreme rays have rank $d-1$ so this bound becomes $\ell\left(\Lambda_{+}(p, e)\right) \leq 3$.

A hyperbolic polynomial is irreducible if it cannot be factored as a product of polynomials of lower degree. If $p$ is hyperbolic with respect to $e$ and is reducible, we can write $p=p_{1}^{m_{1}} \cdots p_{n}^{m_{n}}$ where the $p_{i}$ are the irreducible factors of $p$ and the $m_{i}$ are positive integers. It is straightforward to see that the $p_{i}$ are also hyperbolic with respect to $e$. The hyperbolicity cone corresponding to $p$ is the intersection of the cones corresponding to the $p_{i}$. As such,

$$
\Lambda_{+}(p, e)=\left\{x:(x, x, \ldots, x) \in \Lambda_{+}\left(p_{1}, e\right) \times \cdots \times \Lambda_{+}\left(p_{n}, e\right)\right\}
$$

gives a $\Lambda_{+}\left(p_{1}, e\right) \times \cdots \times \Lambda_{+}\left(p_{n}, e\right)$-lift of $\Lambda_{+}(p, e)$.

The following corollary of Theorem 1.4 summarizes some of the discussion above, and highlights one of very few known obstructions to the existence of lifts using hyperbolicity cones.

\footnotetext{
${ }^{2}$ Self-dual conex cones for which the automorphism group acts transitively on the interior.
} 
Corollary 2.3. Suppose $C$ is a proper convex cone such that for any $N \in \mathbb{N}$ there is a subset $V_{N} \subseteq \operatorname{ext}(C)$ such that $\left|V_{N}\right| \geq N$ and $C$ is $k$-neighborly with respect to $V_{N}$. If $p$ is hyperbolic with respect to e and all the irreducible components of $p$ have degree at most $k$, then $C$ does not have a $\Lambda_{+}(p, e)$-lift.

\section{Technical preliminaries}

In this section we briefly introduce the additional definitions, and basic technical results, needed for our proof of Theorem 1.4.

\subsection{Convex cones and their faces}

The lineality space $\operatorname{Lin}(C)$ of a closed convex cone is $\operatorname{Lin}(C):=C \cap(-C)$, and is the largest subspace contained in $C$. A closed convex cone $C$ is pointed if and only if $\operatorname{Lin}(C)=\{0\}$. A closed convex cone can always be expressed as $C=C^{\prime}+\operatorname{Lin}(C)$ where $C^{\prime}$ is pointed and the sum is direct (for instance we can take $\left.C^{\prime}=C \cap \operatorname{Lin}(C)^{\perp}\right)$.

If $C \subseteq \mathbb{R}^{n}$ is a closed convex cone and $X \subseteq C$, we denote by $F_{C}(X)$ the smallest (inclusionwise) face of $C$ containing $X$. If $x \in C$, we write $F_{C}(x)$ instead of $F_{C}(\{x\})$ for the smallest (inclusion-wise) face of $C$ containing $x$. If $C$ is clear from the context, we omit it from the notation.

The collection of faces of a proper convex cone $C$, partially ordered by inclusion, form a lattice [Bar73]. The lattice operations are

$$
F_{1} \wedge F_{2}=F_{1} \cap F_{2} \quad \text { and } \quad F_{1} \vee F_{2}=F_{C}\left(F_{1} \cup F_{2}\right) .
$$

We summarize some useful properties of this operation for future reference.

Lemma 3.1. Let $C$ be a proper convex cone.

1. If $F$ is a face of $C$ and $F \subsetneq C$ then $\operatorname{dim}(F)<\operatorname{dim}(C)$.

2. If $x \in C$ and $F$ is a face of $C$ then $F_{C}(x)=F$ if and only if $x \in \operatorname{relint}(F)$.

3. If $\lambda>0$ and $x \in C$ then $F_{C}(\lambda x)=F_{C}(x)$.

4. If $x, y \in C$ then $F_{C}(x+y)=F_{C}(x) \vee F_{C}(y)$.

5. If $x_{1}, \ldots, x_{n} \in C$ and $\lambda_{1}, \ldots, \lambda_{n}>0$ then

$$
F_{C}\left(\sum_{i=1}^{n} \lambda_{i} x_{i}\right)=\bigvee_{i=1}^{n} F_{C}\left(\lambda_{i} x_{i}\right)=\bigvee_{i=1}^{n} F_{C}\left(x_{i}\right)=F_{C}\left(\sum_{i=1}^{n} x_{i}\right) .
$$

Proof. The first statement is [Roc15, Corollary 18.1.3]. For the second statement, suppose that $x \in \operatorname{relint}(F)$. Then $F$ is a face of $C$ that contains $x$, so $F_{C}(x) \subseteq F$. Moreover, $x \in F_{C}(x) \cap \operatorname{relint}(F)$ so, by [Roc15, Corollary 18.1.2], $F \subseteq F_{C}(x)$. Now assume that $F_{C}(x)=F$. Then, by [Bar73, Lemma 2.9], $x \in \operatorname{relint}\left(F_{C}(x)\right)=\operatorname{relint}(F)$. The third statement follows from the fact that if $F$ is a face of $C$ then $x \in F$ if and only if $\lambda x \in F$. The fourth statement is a special case of [BC75, Corollary 1]. The fifth statement follows from statements 3 and 4 . 


\subsection{Cone lifts and the factorization theorem}

We begin with the notion of a proper ${ }^{3}$ lift, a slight refinement of Definition 1.1.

Definition 3.2. If $C \subseteq \mathbb{R}^{n}$ and $K \subseteq \mathbb{R}^{d}$ are closed convex cones then $C$ has a proper $K$-lift if $C=\pi(K \cap L)$ where $\pi: \mathbb{R}^{d} \rightarrow \mathbb{R}^{n}$ is a linear map and $L \subseteq \mathbb{R}^{d}$ is a linear subspace that meets the relative interior of $K$.

The factorization theorem [GPT13, Theorem 1] of Gouveia, Parrilo, and Thomas, allows us to reformulate geometric questions about the existence of cone lifts as algebraic questions about the existence of factorizations of certain non-negative operators. Here we state and prove (for completeness) only the direction of the result of Gouveia, Parrilo, and Thomas that we need, and express it in a modified form that is most convenient for our subsequent use.

Theorem 3.3 (Factorization theorem [GPT13, Theorem 1]). If a proper convex cone $C$ has a proper $K_{1} \times \cdots \times K_{m}$-lift then, for $i=1,2, \ldots, m$, there are maps $a_{i}: C^{*} \rightarrow K_{i}^{*}$ and $b_{i}: C \rightarrow K_{i}$ such that $\langle x, y\rangle=\sum_{i=1}^{m}\left\langle b_{i}(x), a_{i}(y)\right\rangle$ for all $(x, y) \in C \times C^{*}$.

Proof. Since $C$ has a proper $K:=K_{1} \times \cdots \times K_{m}$-lift there is a subspace $L$ that meets the relative interior of $K$ such that $C=\pi(K \cap L)$. For each $x \in C$ define $b(x)$ to be an arbitrary choice of element of $K \cap L$ such that $\pi(b(x))=x$.

Since $C=\pi(K \cap L)$ and $L$ meets the relative interior of $K$, it follows from [Roc15, Corollary 16.4.2] that $\pi^{*}\left(C^{*}\right) \subseteq(K \cap L)^{*}=K^{*}+L^{\perp}$. So, for each $y \in C^{*}$, there exists $a(y) \in K^{*}$ and $w(y) \in L^{\perp}$ such that $\pi^{*}(y)=a(y)+w(y)$. Then

$$
\langle x, y\rangle=\langle\pi(b(x)), y\rangle=\left\langle b(x), \pi^{*}(y)\right\rangle=\langle b(x), a(y)+w(y)\rangle=\langle b(x), a(y)\rangle
$$

since $b(x) \in L$ and $w(y) \in L^{\perp}$. If we let the $a_{i}$ and $b_{i}$ be the associated coordinate functions of $a$ and $b$, the result follows.

The following result means we can replace general lifts with proper lifts when we prove Theorem 1.4, allowing us to use the factorization theorem.

Lemma 3.4. Suppose that $C$ is a proper convex cone and there exist a positive integer $m$ and closed convex cones $\tilde{K}_{1}, \tilde{K}_{2}, \ldots, \tilde{K}_{m}$ such that $C$ has a $\tilde{K}_{1} \times \cdots \times \tilde{K}_{m}$-lift and $\ell\left(\tilde{K}_{i}\right) \leq k+1$ for all $i \in[m]$. Then there exist a positive integer $m$ and closed convex cones $K_{1}, K_{2}, \ldots, K_{m}$ such that $C$ has a proper $K_{1} \times \cdots \times K_{m}$-lift and $\ell\left(K_{i}\right) \leq k+1$ for all $i \in[m]$.

Proof. By our assumption on $C$, there is a linear map $\pi$ and a subspace $L$ such that $C=\pi(L \cap$ $\left.\left(\tilde{K}_{1} \times \cdots \times \tilde{K}_{m}\right)\right)$. If $L$ meets the relative interior of $\tilde{K}_{1} \times \cdots \times \tilde{K}_{m}$ we simply take $K_{i}=\tilde{K}_{i}$ for all $i \in[m]$. If $L$ does not intersect the relative interior of $\tilde{K}_{1} \times \cdots \times \tilde{K}_{m}$ then let $F$ denote the minimal face of $\tilde{K}_{1} \times \cdots \times \tilde{K}_{m}$ containing $\left(\tilde{K}_{1} \times \cdots \times \tilde{K}_{m}\right) \cap L$. Since $F$ is a face of $\tilde{K}_{1} \times \cdots \times \tilde{K}_{m}$ it has the form $F_{1} \times \cdots \times F_{m}$ where $F_{i}$ is a face of $\tilde{K}_{i}$ for all $i \in[m]$ [Bar78, Theorem 2]. Moreover, we have that $\ell\left(F_{i}\right) \leq \ell\left(\tilde{K}_{i}\right)$ for all $i \in[m]$. As such, $C$ has a proper $F_{1} \times F_{2} \times \cdots \times F_{m}$-lift and $\ell\left(F_{i}\right) \leq k+1$ for all $i \in[m]$. Taking $K_{i}=F_{i}$ for all $i \in[m]$ completes the proof.

\footnotetext{
${ }^{3}$ The notion of a proper lift of a closed convex cone (from Definition 3.2) is quite distinct from the notion of a proper (i.e., closed, pointed, full-dimensional) convex cone. The distinction between these (standard) uses should be clear from the context.
} 


\subsection{Ramsey numbers}

The key combinatorial result we use is Ramsey's theorem for hypergraphs. We formally state it in language more suited to our application.

Theorem 3.5 ([Ram30, Theorem B]). Let $k, m$, and $n$ be positive integers. There exists a positive integer $R_{k}(m ; n)$ such that whenever $V$ is a set with $|V| \geq R_{k}(m ; n)$ and $f:\left(\begin{array}{l}V \\ k\end{array}\right) \rightarrow[n]$ then there exists $W \subseteq V$ such that $|W|=m$ and $f(S)=f(T)$ for all $S, T \in\left(\begin{array}{c}W \\ k\end{array}\right)$.

In the hypergraph setting we think of $f$ as a coloring of the $k$-uniform hypergraph on vertex set $V$ with $n$ colors, and $W$ as a subset of $m$ vertices of the hypergraph for which all induced hyperedges have the same color. Except in a few very special cases, the numbers $R_{k}(m ; n)$ are not known, although explicit upper bounds are available. For our purposes, we only need the fact that $R_{k}(m ; n)$ is finite for positive integers $n, k$, and $m$.

\section{Generalizing Averkov's lemmas and the proof of Theorem 1.4}

In this section we establish Theorem 1.4 by generalizing the key technical arguments of [Ave19]. Crucial to Averkov's approach is the following simple result about positive semidefinite matrices. In the statement, if $X$ is a positive semidefinite matrix, let $\operatorname{col}(X)$ denote its column space, and interpret the empty sum as zero, i.e., $\sum_{i \in \emptyset} X_{i}=0$.

Lemma 4.1. Suppose $X_{1}, X_{2}, \ldots, X_{\ell} \succeq 0$ and $\operatorname{rank}\left(\sum_{i \in[n]} X_{i}\right)=k$. Then there exists a subset $I \subseteq[n]$ with $|I| \leq k$ such that $\operatorname{col}\left(\sum_{i \in I} X_{i}\right)=\operatorname{col}\left(\sum_{i \in[n]} X_{i}\right)$.

We note that Averkov states the conclusion in an equivalent form as $\sum_{i \in I} \operatorname{col}\left(X_{i}\right)=\sum_{i \in[n]} \operatorname{col}\left(X_{i}\right)$. The statement given here suggests, more clearly, the generalization we require.

Lemma 4.1 can be generalized to the setting in which the rank is replaced with the largest length of a chain of nonempty faces (minus one), and the column space is replaced with the minimal face containing a point of the convex cone. In the statement and proof of Lemma 4.2 we interpret the empty sum as zero, i.e., $\sum_{i \in \emptyset} x_{i}=0$.

Lemma 4.2. Let $K$ be a closed convex cone and suppose that $x_{1}, x_{2}, \ldots, x_{n} \in K$ are such that $\sum_{i=1}^{n} x_{i} \in \operatorname{relint}(K)$. Then there exists $I \subseteq[n]$ with $|I| \leq \ell(K)-1$ such that $F\left(\sum_{i \in I} x_{i}\right)=$ $F\left(\sum_{i \in[n]} x_{i}\right)=K$.

Proof. First, we will prove the result under the assumption that $K$ is pointed. We argue by induction on $\ell(K)$. For the base case, consider a closed pointed convex cone with $\ell(K)=1$. The only possibility is $K=\{0\}$. In this case if $x_{1}, \ldots, x_{n} \in K$ we can choose $I=\emptyset$ so that $|I|=0=\ell(\{0\})-1$ and $F\left(\sum_{i \in I} x_{i}\right)=F(0)=\{0\}=K$.

Assume the statement holds for all closed pointed convex cones $K$ with $\ell(K) \leq k$, for some positive integer $k$. Consider a closed pointed convex cone $C$ with $\ell(C)=k+1$. Let $x_{1}, \ldots, x_{n} \in C$ be such that $F\left(x_{1}+\cdots+x_{n}\right)=C$. Let $I \subseteq[n]$ be an inclusion-wise minimal subset of $[n]$ such that $F\left(\sum_{i \in I} x_{i}\right)=C$. Choose some $j \in I$ and observe that $x_{j} \notin F\left(\sum_{i \in I \backslash\{j\}} x_{i}\right)$ (by minimality of $I)$ and so $F\left(\sum_{i \in I \backslash\{j\}} x_{i}\right)$ is strictly contained in $C$. Hence,

$$
\ell\left(F\left(\sum_{i \in I \backslash\{j\}} x_{i}\right)\right) \leq \ell(C)-1=k .
$$

Since $F\left(\sum_{i \in I \backslash\{j\}} x_{i}\right)$ is closed, convex, and pointed, by the induction hypothesis, there is some $I^{\prime} \subseteq I \backslash\{j\}$ such that $\left|I^{\prime}\right| \leq k-1$ and

$$
F\left(\sum_{i \in I^{\prime}} x_{i}\right)=F\left(\sum_{i \in I \backslash\{j\}} x_{i}\right) .
$$


Then, by Lemma 3.1,

$$
\begin{aligned}
F\left(x_{j}+\sum_{i \in I^{\prime}} x_{i}\right) & =F\left(x_{j}\right) \vee F\left(\sum_{i \in I^{\prime}} x_{i}\right) \\
& =F\left(x_{j}\right) \vee F\left(\sum_{i \in I \backslash\{j\}} x_{i}\right)=F\left(\sum_{i \in I} x_{i}\right)=C .
\end{aligned}
$$

Since $I$ is minimal with this property and $I^{\prime} \cup\{j\} \subseteq I$ we must have that $I^{\prime} \cup\{j\}=I$. It then follows that $|I|=\left|I^{\prime}\right|+1 \leq k$, as required.

If $K$ is not pointed, then $K=K^{\prime}+\operatorname{Lin}(K)$ where $K^{\prime}$ is pointed and the sum is direct. Furthermore, $F^{\prime}$ is a face of $K^{\prime}$ if and only if $F^{\prime}+\operatorname{Lin}(K)$ is a face of $K$. If $x=x^{\prime}+z$ with $x^{\prime} \in K^{\prime}$ and $z \in \operatorname{Lin}(K)$ then $x \in \operatorname{relint}(K)$ if and only if $x^{\prime} \in \operatorname{relint}\left(K^{\prime}\right)$.

Now, let $x_{i}=x_{i}^{\prime}+z_{i}$ be decompositions of each of the $x_{i}$ so that $x_{i}^{\prime} \in K^{\prime}$ and $z_{i} \in \operatorname{Lin}(K)$ for all $i$. By assumption, $\sum_{i=1}^{n} x_{i} \in \operatorname{relint}(K)$ and so $\sum_{i=1}^{n} x_{i}^{\prime} \in \operatorname{relint}\left(K^{\prime}\right)$. Since the lemma holds for the pointed case, there exists $I \subseteq[n]$ with $|I| \leq \ell\left(K^{\prime}\right)-1=\ell(K)-1$ such that $\sum_{i \in I} x_{i}^{\prime} \in \operatorname{relint}\left(K^{\prime}\right)$. Then $\sum_{i \in I} x_{i} \in \operatorname{relint}(K)$ as required.

Averkov's main lemma is the following result, which relies crucially on Lemma 4.1 for its proof.

Lemma 4.3. Let $m$ be a positive integer and let $S$ denote a finite set with cardinality at least $k$. Suppose that, for each $i \in[m]$, there are maps $a_{i}:\left(\begin{array}{l}S \\ k\end{array}\right) \rightarrow \mathcal{S}_{+}^{k}$ and $b_{i}: S \rightarrow \mathcal{S}_{+}^{k}$ such that

$$
\sum_{i=1}^{m}\left\langle a_{i}(T), b_{i}(s)\right\rangle=0 \quad \Longleftrightarrow s \in T .
$$

Then $|S|<R_{k}\left(k+1 ;(k+1)^{m}\right)$.

We can use essentially the same argument as Averkov to establish the following analogue of Lemma 4.3, once we replace column spaces by minimal faces and rank with the largest length of a chain of nonempty faces (minus one). We recover Averkov's lemma as a special case by noting that the cone $\mathcal{S}_{+}^{k}$ is self-dual and satisfies $\ell\left(\mathcal{S}_{+}^{k}\right)=k+1$.

Lemma 4.4. Let $m$ be a positive integer and let $K_{1}, K_{2}, \ldots, K_{m}$ be closed convex cones. Let $S$ denote a finite set with cardinality at least $k$. Suppose that, for each $i \in[m]$, there are maps $a_{i}:\left(\begin{array}{l}S \\ k\end{array}\right) \rightarrow K_{i}^{*}$ and $b_{i}: S \rightarrow K_{i}$ such that

$$
\sum_{i=1}^{m}\left\langle a_{i}(T), b_{i}(s)\right\rangle=0 \quad \Longleftrightarrow s \in T .
$$

If $\ell\left(K_{i}\right) \leq k+1$ for $i=1,2, \ldots, m$ then $|S|<R_{k}\left(k+1 ;(k+1)^{m}\right)$.

Proof. For each $T \subseteq S$ and each $i \in[m]$ define

$$
b_{T, i}:=\sum_{t \in T} b_{i}(t) \in K_{i} \quad \text { and } \quad d_{T, i}:=\ell\left(F\left(b_{T, i}\right)\right) .
$$

For each $T \in\left(\begin{array}{l}S \\ k\end{array}\right)$, we assign 'color' $\left(d_{T, 1}, \ldots, d_{T, m}\right)$ to the set $T$. Since $\ell\left(K_{i}\right) \leq k+1$, then the same is true for any nonempty face of $K_{i}$, and so $d_{T, i} \in\{0,1, \ldots, k\}$ for each $T$ and $i$. As such, we are coloring with at most $(k+1)^{m}$ colors.

Seeking a contradiction, let us assume that $|S| \geq R_{k}\left(k+1 ;(k+1)^{m}\right)$. Then, by the definition of the Ramsey number, there exists $W \subseteq S$ with $|W|=k+1$ such that all $k$-element subsets of $W$ have the same color $\left(d_{1}, d_{2}, \ldots, d_{m}\right)$. More explicitly, for all $T \subset W$ with $|T|=k$ we have $\left(d_{T, 1}, \ldots, d_{T, m}\right)=\left(d_{1}, \ldots, d_{m}\right)$. 
Claim For each $i \in[m]$ and each $T \subset W$ such that $|T|=k$, we have that $F\left(b_{T, i}\right)=F\left(b_{W, i}\right)$. If this were not the case, then there exists such an $i \in[m]$ and $T \subset W$ with $|T|=k$ such that $F\left(b_{W, i}\right)$ strictly contains $F\left(b_{T, i}\right)$. By Lemma 4.2 , there is a subset $T^{\prime} \subseteq W$ with $\left|T^{\prime}\right| \leq \ell\left(F\left(b_{W, i}\right)\right)-1 \leq k$ such that $F\left(\sum_{t^{\prime} \in T^{\prime}} b_{i}\left(t^{\prime}\right)\right)=F\left(b_{W, i}\right)$. By adding elements of $W$ (if necessary) we can form a set $T^{\prime \prime}$ such that $T^{\prime} \subseteq T^{\prime \prime} \subset W$ and $\left|T^{\prime \prime}\right|=k$ such that

$$
F\left(\sum_{t^{\prime \prime} \in T^{\prime \prime}} b_{i}\left(t^{\prime \prime}\right)\right) \supseteq F\left(\sum_{t^{\prime} \in T^{\prime}} b_{i}\left(t^{\prime}\right)\right)=F\left(b_{W, i}\right) .
$$

One the one hand, since all $k$-element subsets of $W$ have the same 'color', $d_{i}=d_{T^{\prime \prime}, i} \geq \ell\left(F\left(b_{W, i}\right)\right)$. On the other hand, since $F\left(b_{W, i}\right)$ strictly contains $F\left(b_{T, i}\right)$ we have that $\ell\left(F\left(b_{W, i}\right)\right)>\ell\left(F\left(b_{T, i}\right)\right)=d_{i}$. This contradiction establishes the claim.

With the claim established, we write $W=T \cup\{s\}$ where $|T|=k$ and $s \notin T$. By assumption on $a_{i}$ and $b_{i}$, we have that

$$
\sum_{i=1}^{m}\left\langle a_{i}(T), b_{i}(t)\right\rangle=0 \quad \text { for all } t \in T .
$$

Since $a_{i}(T) \in K_{i}^{*}$ and $b_{i}(t) \in K_{i}$ we can conclude that

$$
\left\langle a_{i}(T), b_{i}(t)\right\rangle=0 \quad \text { for all } t \in T \text { and all } i \in[m] .
$$

But then

$$
\left\langle a_{i}(T), \sum_{t \in T} b_{i}(t)\right\rangle=0 .
$$

In particular, for each $i \in[m]$ the linear functional defined by $a_{i}(T)$ vanishes on the face

$$
F\left(\sum_{t \in T} b_{i}(t)\right)=F\left(b_{T, i}\right)=F\left(b_{W, i}\right)
$$

where the last equality is the claim. But then, even though $s \notin T$, we have that

$$
0=\left\langle a_{i}(T), \sum_{w \in W} b_{i}(w)\right\rangle=\left\langle a_{i}(T), b_{i}(s)+\sum_{t \in T} b_{i}(t)\right\rangle=\left\langle a_{i}(T), b_{i}(s)\right\rangle
$$

for all $i \in[m]$. This contradicts the fact that $a$ and $b$ satisfy $0=\sum_{i=1}^{m}\left\langle a_{i}(T), b_{i}(s)\right\rangle$ if and only if $s \in T$. It then follows that $|S|<R_{k}\left(k+1 ;(k+1)^{m}\right)$.

Following Averkov, we combine Lemma 4.3 with the factorization theorem of Gouveia, Parrilo, and Thomas (Theorem 3.3) to turn the statement about the structure of maps $a_{i}$ and $b_{i}$ into a statement about lifts of convex cones. We briefly repeat the argument here, in our setting, to make the paper more self-contained.

Proposition 4.5. Suppose that a proper convex cone $C \subseteq \mathbb{R}^{n}$ has a proper $K_{1} \times \cdots \times K_{m}$-lift where $\ell\left(K_{i}\right) \leq k+1$ for $i \in[m]$. If $C$ is $k$-neighborly with respect to some finite set $V \subseteq \operatorname{ext}(C)$ then $|V|<R_{k}\left(k+1 ;(k+1)^{m}\right)$.

Proof. Since $C$ is $k$-neighborly with respect to $V$, for each $k$-element subset $W \in\left(\begin{array}{l}V \\ k\end{array}\right)$ there is $f_{W} \in C^{*}$ such that $\left\langle f_{W}, w\right\rangle$ vanishes if $w \in W$ and is positive if $w \in V \backslash W$. Since $C$ has a proper $K_{1} \times \cdots \times K_{m}$ lift, by the factorization theorem (Theorem 3.3) we know that for $i=1,2, \ldots, m$ 
there exist maps $\tilde{a}_{i}: C^{*} \rightarrow K_{i}^{*}$ and $\tilde{b}_{i}: C \rightarrow K_{i}$ such that $\langle f, x\rangle=\sum_{i=1}^{m}\left\langle\tilde{a}_{i}(f), \tilde{b}_{i}(x)\right\rangle$ for all $f \in C^{*}$ and all $x \in C$. Define $a_{i}:\left(\begin{array}{c}V \\ k\end{array}\right) \rightarrow K_{i}^{*}$ by $a_{i}(W)=\tilde{a}_{i}\left(f_{W}\right)$ and $b_{i}: V \rightarrow K_{i}$ by $b_{i}(w)=\tilde{b}_{i}(w)$. Then $\left\langle f_{W}, w\right\rangle=\sum_{i=1}^{m}\left\langle a_{i}(W), b_{i}(w)\right\rangle$ which vanishes when $w \in W$ and is positive when $w \in V \backslash W$. It follows from Lemma 4.4 that $|V|<R_{k}\left(k+1 ;(k+1)^{m}\right)$.

Theorem 1.4 is a straightforward consequence of Proposition 4.5.

Proof of Theorem 1.4. By assumption, there exists some finite $V \subset \operatorname{ext}(C)$ such that $|V| \geq R_{k}(k+$ $1 ;(k+1)^{m}$ ) and $C$ is $k$-neighborly with respect to $V$. Then by the contrapositive of Proposition 4.5 it follows that $C$ does not have a proper $K_{1} \times \cdots \times K_{m}$ lift with $\ell\left(K_{i}\right) \leq k+1$ for all $i \in[m]$. It follows from Lemma 3.4 that $C$ does not have a (possibly improper) $\tilde{K}_{1} \times \cdots \times \tilde{K}_{m}$-lift with $\ell\left(\tilde{K}_{i}\right) \leq k+1$ for all $i \in[m]$, completing the proof.

\section{Discussion}

In this paper we have shown that for a convex cone $C$, having a certain neighborliness property is an obstruction to having a $K$-lift where $K$ is a Cartesian product of cones all of which only have short chains of faces. Our argument is a fairly direct generalization of an argument of Averkov showing that the same neighborliness property is an obstruction to having an $\left(\mathcal{S}_{+}^{k}\right)^{m}$-lift.

Although we only stated qualitative results about the non-existence of lifts, the approach taken in this paper could be made quantitative in the following sense. If $C$ is $k$-neighborly with respect to a finite set $V$ and $\ell\left(K_{i}\right) \leq k+1$ for all $i$, then Theorem 1.4 does not rule out the possibility that $C$ has a $K_{1} \times \cdots \times K_{m}$-lift. However one could, in principle, extract a lower bound on the number of factors $m$ required in any such lift in terms of $k$ and $|V|$. Unfortunately, this gives very weak lower bounds, since the Ramsey numbers $R_{k}\left(k+1 ;(k+1)^{m}\right)$ grow extremely fast (and so the implied lower bounds on $m$ grows extremely slowly). For convex cones that do have $K_{1} \times \cdots \times K_{m}$-lifts where $\ell\left(K_{i}\right) \leq k+1$ for all $i$, it would be very interesting to develop general techniques to establish stronger lower bounds on $m$, the number of factors. In the case $k=1$ this reduces to the study of lower bounds on the linear programming extension complexity of a polyhedral cone.

This paper gives some of the first results about non-existence of $K$-lifts where $K$ is a hyperbolicity cone. Currently the only other such result is a lower bound on the size of PSD lifts of convex semialgebraic sets, based on quantifier elimination [GPT13], that can be generalized directly to the setting of hyperbolicity cones. Fawzi and Safey El Din [FSED18] strengthen the quantifier elimination-based bounds in the case of PSD lifts by exploiting connections with the algebraic degree of semidefinite programming [NRS10]. It would be interesting to develop a similar approach to devise lower bounds on natural notions of complexity for lifts using hyperbolicity cones.

Acknowledgments I would like to thank Hamza Fawzi for encouraging and insightful comments

related to this work, Venkat Chandrasekaran for introducing me to the notion of the length of the longest chain of nonempty faces of a convex cone, and the anonymous referees for their careful reading and very helpful comments.

\section{References}

[AB18] N. Amini and P. Brändén. Non-representable hyperbolic matroids. Advances in Mathematics, 334:417-449, 2018. 
[AHMR88] J. Agler, W. Helton, S. McCullough, and L. Rodman. Positive semidefinite matrices with a given sparsity pattern. Linear algebra and its applications, 107:101-149, 1988.

[AM19] A. A. Ahmadi and A. Majumdar. DSOS and SDSOS optimization: more tractable alternatives to sum of squares and semidefinite optimization. SIAM Journal on Applied Algebra and Geometry, 3(2):193-230, 2019.

[Ave19] G. Averkov. Optimal size of linear matrix inequalities in semidefinite approaches to polynomial optimization. SIAM Journal on Applied Algebra and Geometry, 3(1):128$151,2019$.

[Bar73] G. P. Barker. The lattice of faces of a finite dimensional cone. Linear Algebra and its Applications, 7(1):71-82, 1973.

[Bar78] G. P. Barker. Perfect cones. Linear Algebra and its Applications, 22:211-221, 1978.

[BC75] G. P. Barker and D. Carlson. Cones of diagonally dominant matrices. Pacific Journal of Mathematics, 57(1):15-32, 1975.

[BSV17] G. Blekherman, R. Sinn, and M. Velasco. Do sums of squares dream of free resolutions? SIAM Journal on Applied Algebra and Geometry, 1(1):175-199, 2017.

[BTN01] A. Ben-Tal and A. Nemirovski. Lectures on modern convex optimization: analysis, algorithms, and engineering applications, volume 2. SIAM, 2001.

[CS16] V. Chandrasekaran and P. Shah. Relative entropy relaxations for signomial optimization. SIAM Journal on Optimization, 26(2):1147-1173, 2016.

[Faw18] H. Fawzi. On representing the positive semidefinite cone using the second-order cone. Mathematical Programming, pages 1-10, 2018.

[FSED18] H. Fawzi and M. Safey El Din. A lower bound on the positive semidefinite rank of convex bodies. SIAM Journal on Applied Algebra and Geometry, 2(1):126-139, 2018.

[Går59] L. Gårding. An inequality for hyperbolic polynomials. Journal of Mathematics and Mechanics, pages 957-965, 1959.

[GJSW84] R. Grone, C. R. Johnson, E. M. Sá, and H. Wolkowicz. Positive definite completions of partial Hermitian matrices. Linear algebra and its applications, 58:109-124, 1984.

[GPT13] J. Gouveia, P. A. Parrilo, and R. R. Thomas. Lifts of convex sets and cone factorizations. Mathematics of Operations Research, 38(2):248-264, 2013.

[IDW16] S. Iliman and T. De Wolff. Amoebas, nonnegative polynomials and sums of squares supported on circuits. Research in the Mathematical Sciences, 3(1):9, 2016.

[IL17] M. Ito and B. F. Lourenço. A bound on the Carathéodory number. Linear Algebra and its Applications, 532:347-363, 2017.

[KW08] G. Kalai and A. Wigderson. Neighborly embedded manifolds. Discrete \& Computational Geometry, 40(3):319-324, 2008.

[LP18] M. Liu and G. Pataki. Exact duals and short certificates of infeasibility and weak infeasibility in conic linear programming. Mathematical Programming, 167(2):435-480, 2018. 
[NRS10] J. Nie, K. Ranestad, and B. Sturmfels. The algebraic degree of semidefinite programming. Mathematical Programming, 122(2):379-405, 2010.

[Nui69] W. Nuij. A note on hyperbolic polynomials. Mathematica Scandinavica, 23(1):69-72, 1969.

[Pat13] G. Pataki. Strong duality in conic linear programming: facial reduction and extended duals. In Computational and analytical mathematics, pages 613-634. Springer, 2013.

[Ram30] F. P. Ramsey. On a problem of formal logic. Proceedings of the London Mathematical Society, 2(1):264-286, 1930.

[Ren06] J. Renegar. Hyperbolic programs, and their derivative relaxations. Foundations of Computational Mathematics, 6(1):59-79, 2006.

[Roc15] R. T. Rockafellar. Convex analysis. Princeton University Press, 2015.

[VA15] L. Vandenberghe and M. S. Andersen. Chordal graphs and semidefinite optimization. Foundations and Trends@ in Optimization, 1(4):241-433, 2015.

[WM13] H. Waki and M. Muramatsu. Facial reduction algorithms for conic optimization problems. Journal of Optimization Theory and Applications, 158(1):188-215, 2013. 